\title{
Mitigating the Attraction Effect with Visualizations
}

\author{
Evanthia Dimara, Gilles Bailly, Anastasia Bezerianos, and Steven Franconeri
}

\begin{abstract}
Human decisions are prone to biases, and this is no less true for decisions made within data visualizations. Bias mitigation strategies often focus on the person, by educating people about their biases, typically with little success. We focus instead on the system, presenting the first evidence that altering the design of an interactive visualization tool can mitigate a strong bias - the attraction effect. Participants viewed 2D scatterplots where choices between superior alternatives were affected by the placement of other suboptimal points. We found that highlighting the superior alternatives weakened the bias, but did not eliminate it. We then tested an interactive approach where participants completely removed locally dominated points from the view, inspired by the elimination by aspects strategy in the decision-making literature. This approach strongly decreased the bias, leading to a counterintuitive suggestion: tools that allow removing inappropriately salient or distracting data from a view may help lead users to make more rational decisions.
\end{abstract}

Index Terms-Decision making, cognitive bias, bias alleviation, bias mitigation, debiasing, information visualization, attraction effect.

\section{INTRODUCTION}

We often misweigh information based on its salience, or how congruent it is with our fears or desires. People often misjudge probabilities, like the odds of having breast cancer after a positive mammography [4]. Or once we have formed a belief, we tend to overweigh information that confirms that belief [66]. These are cognitive biases. According to Pohl's definition [75], cognitive biases are phenomena that involuntarily deviate from a normative model and the normal course of information processing, in a predictable way (as opposed to judgement that is unpredictably inaccurate, for example due to misunderstanding information). Cognitive biases differ from perceptual biases in that they exist even after veridically perceiving information. To measure a cognitive bias, researchers use synthetic tasks in constrained environments [24]. But biases also appear in critical real-world situations such as in the national security [77] or medical [61] domains, even for intelligent and open-minded decision makers [92] (though see [33] for a skeptical view of the ecological validity of many claimed biases).

Cognitive bias mitigation methods, known also as debiasing, focus primarily on educating the decision maker, (e.g., through statistics training [30]). They have shown limited or only temporary success [75], and are ineffective $[3,27,49,79]$ even in critical domains such as medicine $[19,34]$ or policy making [66]. A more rarely implemented approach is to debias the environment instead of the decision maker [53]. Previous studies that use this approach altered the design of textual information $[40,53]$, but did not consider the use of visualizations.

The visualization community has explored the idea that cognitive biases may arise during visual analysis [24, 89,98]. Dimara et al. [22] found that in scatterplots, decisions among optimal choices (points in the 2D space of the scatterplot) were influenced by the presence of suboptimal choices, replicating the attraction effect. But when past research has explored ways of mitigating such biases with visualizations, results have remained inconclusive [24]. For example, Micallef et al. [59] explored whether Euler diagrams could improve probabilistic reasoning to mitigate the base rate fallacy [4], and Boy et al. [8] tested whether anthropomorphized icons would decrease an impulse to dehumanize statistical data about suffering populations [47]. Sur-

- Evanthia Dimara is with ISIR and INRIA. E-mail: evanthia.dimara@gmail.com.

- Gilles Bailly is with ISIR. E-mail: gilles.bailly@upmc.fr.

- Anastasia Bezerianos Univ. Paris-Sud \& CNRS (LRI), Inria, and Univ. Paris-Saclay. E-mail: anastasia.bezerianos@lri.fr.

- Steven Franconeri is with with Northwestern Univ. E-mail: franconeri@northwestern.edu

Manuscript received xx xxx. 201x; accepted xx xxx. 201x. Date of Publication xx xxx. 201x; date of current version xx xxx. 201x. For information on obtaining reprints of this article, please send e-mail to: reprints@ieee.org. Digital Object Identifier: $x x . x x x x / T V C G .201 x . x x x x x x x$ prisingly, neither study found evidence for the biases in the first place (such replication failures also occur in the primary literature [44]), and, consequently, if a visualization can alleviate it.

In this paper we take inspiration from the debiasing method of altering the environment of the decision maker [53] by using interactive visualizations. We focus on mitigating the attraction effect bias which has been reliably detected in visualizations in past work [22]. We replicate the setup of that original study, across two studies, and contrast it to interactive displays that alter the decision making environment. In the first visual approach, we explore if highlighting optimal choices could help decision makers focus on the important information while ignoring distracting choices. In the second interactive approach, we explore a stronger manipulation where participants delete suboptimal datapoints from the display, encouraging a decision strategy known as "elimination by aspects" [86].

\section{BACKGROUND}

We next review methods for debiasing decision makers, and connect these debiasing approaches to visualization research.

\subsection{Debias the judge}

Most techniques that have been employed to mitigate cognitive biases appear so far to be rather ineffective [3,27, 49,79]. Most of them focus on how to improve decision making by improving a participant's education or by increasing their motivation.

\subsubsection{Improve education}

"Training in rules" techniques examine the benefits of formal training in economics (e.g., normative theory) [54], social and natural sciences [56], and statistics (e.g., law of small numbers) often by combining abstract rules for normative reasoning with concrete examples $[13,30]$. Unfortunately, these techniques require extensive training, and the improvements that they bring tend to weaken over time [31].

Stronger forms of training can be effective. Computer games and simulations [34] provide immediate feedback, structured learning environments, and tailored instructions based on performance $[16,26,58,60]$. They have proven useful in reducing the fundamental attribution error, bias blind spot, and confirmation bias $[16,60]$, after the session and even eight weeks later. Computer games appear more effective than videos probably because they provide personalized feedback and hands-on experience [60].

Other debiasing techniques teach thinking strategies. In "consider the opposite" decision makers are asked to consider evidence for alternatives in order to widen their sample of evidence, allowing to reduce biases such as overconfidence, hindsight or anchoring [3,64]. This technique, though, can backfire since considering alternatives overwhelms cognitive capacity - when people were asked to list multiple ways in which past events could have turned out, the additional load actually amplifies some biases [79]. 


\subsubsection{Increase motivation}

Other debiasing techniques attempt to increase the participant's motivation to solve a task, typically through reward incentives or accountability. Financial incentives can indeed improve performance for tasks that require more effort [11]. For example, for decisions under uncertainty, one study showed that incentives did not affect mean performance, but they did reduce variance in responses [11], suggesting a more careful and consistent strategy.

However, these incentives typically fail to improve performance when the skills of the participant underlie the bias rather than their level of effort. For example, Fischhoff et al. [28] showed that asking people to wager actual money based on their confidence levels did not manage to mitigate overconfidence. The automatic nature of most cognitive biases may render people unresponsive to incentives, merely motivating them to perform their "suboptimal behavior with more enthusiasm" [3].

Making people accountable for their decisions can also motivate them to put more effort. The assumption behind accountability is that people expected to justify their responses to others would anticipate possible flaws in their reasoning [9]. However, this technique requires the ability to recognize mistakes. Furthermore, accountability can itself bias responses to be more favorable to the audience of interest [9].

\subsection{Rely less on the judge}

Another way to reduce cognitive biases is to rely less on individual human judgment, relying instead on computational solutions or by aggregating multiple individual human judgments.

\subsubsection{Add computation aid}

Actuarial methods [21] — incorporating statistical models based on past data - can be effective. In some clinical judgments that systematically suffer from judgment inconsistencies [21], automated decision support systems (DSS) can run consistency checks (e.g., on attribute weights or probabilities) [48]. They can also incorporate normative algorithms into the decision making process that would be difficult or impossible for human decision makers to compute [20].

However, automation can trigger new cognitive biases when experts over-rely on recommendations from automated systems, even when there are clear indications of disconfirming evidence (e.g., pilots accept significantly sub-optimal flight plans from autopilot systems) [20].

\subsubsection{Encourage group decisions}

One solution to debias critical decisions is to rely on the "wisdom of crowds", as has been implemented for cancer diagnosis, and financial forecast [65]. Each member of a group forms a judgment independently, and a final decision is computed from the aggregated judgments [42], making suboptimal individual strategies less influential [76, 83]. Moreover, there is evidence that making financial choices within a social network can help older adults to overcome their biases in financial risk taking (e.g., to choose retirement savings plans) [96]. There are also cases where deliberation and discussion can improve collective wisdom for responses to general knowledge questions (e.g., what is the height of the Eiffel Tower) [65].

In other cases of members forming judgments during a group discussion, individual biases can be amplified within the final decision [74], Group interactions often trigger other cognitive biases where individuals shape judgments to be favorable to the group [10,29], and group decisions are often biased towards conformity or polarization [62].

\subsection{Debias the environment instead of the judge}

A debiasing approach that has received less attention is to "debias the environment instead of the judge" [53]. Some of these techniques force a slower, more analytical process. In the medical domain, for instance, checklists, deliberate practice, and immediate feedback are suggested to help clinicians avoid diagnostic biases [34], though there is little to no empirical data to demonstrate notable improvement in decision making performance $[19,34]$.

Slowing people down is also possible by presenting information in a disfluent format, which can reduce confirmation bias [40]. For example, jurors gave fewer confirmatory verdicts when reading a summary of a crime which required processing difficulty [40], though it is not clear that this change led to an objectively better decision.

Another tactic is to force people to consider information in isolation from its context, versus in the comparison with other information. In one study where participants distinguished fictional diseases described in text format, one group of participants read about each disease separately from each other, while another group saw simultaneously juxtaposed information from both, which allowed them to highlight the distinctive features of each other. The juxtaposed representation led to more accurate judgments [53].

\subsection{Summary}

Decision making strategies have been divided into 'fast' processes that rely on automatic associations and tend to carry more biases, vs. 'slow' procedural processes that tend to be less biased [50]. Debiasing the judge through education in 'slow' processes can be difficult, as training often does not stick, or transfer to new problems. Relying less on the judge, through computational aids or group decisions, can lead to mixed success. Increasing motivation and incentives can decrease variability in responses, but does not decrease biases. This may be because if a judge only has access to 'fast' processes in the first place, more motivation may improve their attention and engagement, but cannot switch them to a less biased process. Thus, we hope to find success with the strategy of debiasing the environment, by altering the design of a visualization display to force the judge to take a slower and more deliberate approach that is constrained by the task environment itself.

\subsection{Connection to visualization research}

The visualization community has attempted to design and test visual displays that help viewers avoid misunderstandings and reasoning errors in data displays. Some studies demonstrate that misleading designs can cause viewers to incorrectly perceive visualized data [71]. Other studies find that alternative visualization designs $[2,36,68]$, or the use of visualizations instead of text [84] can improve probability estimations. Improving performance in these domains can be difficult [17], even for animated displays [45]. Errors in applying probability or logic rules are often associated with many documented cognitive biases.

Some studies focused explicitly on cognitive bias detection, demonstrating the existence of well known cognitive biases, such as anchoring effects or shifts in decision criteria [14,87,97], the attraction effect [22], or overconfidence effects [2], but do not test designs for mitigating these biases. Some studies evaluated promising design interventions, but did not replicate the original biases $[8,59]$. Others suggest solutions for mitigating cognitive biases by training the viewers to avoid them. For example, we tend to see patterns in noise (e.g., the clustering illusion [51]), a perceptual bias known as pareidolia or apophenia [88]. Because this bias might occur in data visualizations as well, it could be useful to contrast datasets containing signal to other datasets constructed from pure noise [93]. One might envision such training being added to visual analysis systems to mitigate cognitive biases. In summary, previous work within InfoVis shows that cognitive biases affect visualization systems, and while many studies attempt to reveal and improve some reasoning errors, cognitive bias alleviation is challenging.

To alleviate biases, visualization research might seek inspiration from attempts from other domains. The most effective techniques seem to be the educational interventions of Sect. 2.1, video-game training in particular [16]. However, their experimental design did not test multidisciplinary contexts or long-term transfer of knowledge. It is unclear whether a data analyst who successfully solved a confirmation bias puzzle can improve her reasoning during actual visual analysis, as transfer of expertise across examples can be difficult [32]. Perhaps more importantly, extensive training is not always feasible for visualization users, though short-term prescriptions such as "consider the opposite" might inspire the development of visualization systems that encourage analysts to generate alternative hypotheses.

The approach of relying less on the judge, where human judgment is aided by computational methods or group decisions, also remains promising. Similarly, visualization systems can incorporate computation support or encourage collaboration among analysts to reduce 
cognitive biases. For example, collaborative analysis platforms running on shared displays (e.g., [46]) could promote collective wisdom, and if we enhance them with ways to monitor individual and group decisions they could potentially reduce biases present in collaboration.

Our experiments focus on the design-based debiasing approach of transforming the environment of the decision maker. The studies of Sect. 2.3 provide evidence that restructuring the way information is presented can mitigate some cognitive biases. Nevertheless, those studies examined only textual representation formats $[40,53]$, and, although visualizations have been suggested as promising interventions for improving rational reasoning [98], we know of no empirical evidence that visualization systems can mitigate cognitive biases. The next sections will focus on an empirical investigation of how a visualization system could mitigate a known cognitive bias: the attraction effect.

\section{The Attraction Effect}

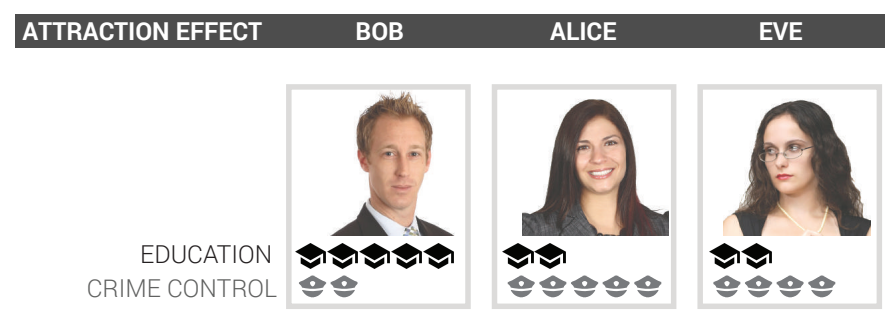

Fig. 1. Attraction effect in elections (example from [22]): Bob has an excellent education plan, while Alice is very strong in crime control. The addition of Eve, a candidate similar but inferior to Alice, raises Alice's attractiveness as a candidate. (Photos Benjamin Miller, FSP Standard License, icons by Ivan Boyco, CC-BY license)

The attraction effect is one of the hundreds of cognitive biases observed in psychology and behavioral economics research [24]. We focus on mitigating the attraction effect because it has been observed in many real-world contexts, including purchase decisions or voting. For example, O'Curry and Pitts [67] showed how the attraction effect can influence voters in a "real world" setup, such as the 1992 U.S. Presidential elections. Recently, evidence shows that it also occurs in scatterplot visualizations [22].

In this section, we first describe the attraction effect and how it was detected in visualizations [22], to motivate the design of the two studies presented in this paper that investigate ways to alleviate this bias using different visualization designs.

\subsection{What is the attraction effect}

Definition. The attraction effect, or decoy effect, is a cognitive bias known primarily from marketing research, as it can strongly affect consumer choices $[43,82]$. If people are deciding between two products ("target" and "competitor"), a third product ("decoy") that is close to the target but objectively suboptimal to both attributes, can make the target look more attractive. Assume two political candidates (Fig. 1). Bob with a solid education plan but not for crime control. And Alice with a good strategy for crime control but not for education. These candidates are uncomparable as both can be good choices depending on how you weigh each dimension. Adding Eve that is similar to Alice (the target), but slightly worse (her crime control strategy is not as good) attracts the decision maker's choice towards Alice [67].

Terminology. To explain the three alternative choices (target, competitor and decoy) we revisit terminology from Dimara et al. [22].

- An alternative dominates another if "it is strictly superior in one attribute and superior or equal in all others".

- An alternative is dominated "within a set of alternatives if there is at least one alternative that dominates it".

- An alternative is asymmetrically dominated " within a set of alternatives if it is dominated by at least one alternative, but is not dominated by at least one other". In the political candidates example, Eve is asymmetrically dominated by Alice, but not Bob.

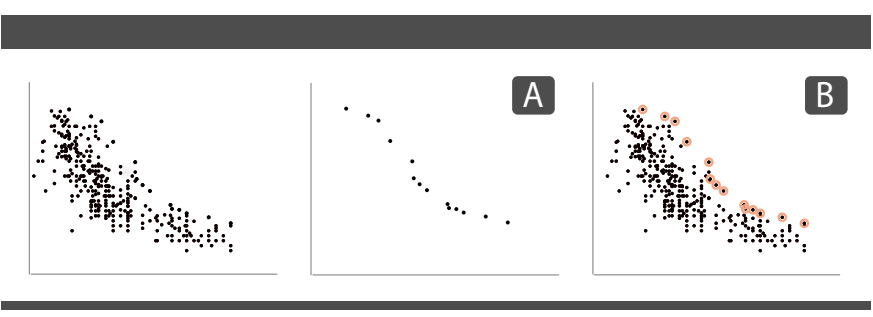

Fig. 2. Possible techniques to mitigate the attraction effect in scatterplots: remove the dominated points $(A)$, indicate visually the Pareto front $(B)$.

- We call a set of alternatives formally uncomparable if no one dominates the other (for example, Bob and Alice).

- The Pareto front is the set of formally uncomparable alternatives [69] (highlighted in orange Fig. 2 B). The optimal alternative in a Pareto front is a matter of personal choice.

- In a typical attraction effect experiment [43], the decision task involves three alternatives, one that is asymmetrically dominated, the decoy, and two formally uncomparable alternatives: the target that dominates the decoy and the competitor that does not dominate the decoy. In other words, both the target and competitor are optimal choices (thus part of the Pareto front) and lead to the maximal payoff.

Findings. When the decoy is absent, participants choose between the two formally uncomparable alternatives according to their personal preferences. If the decoy is present, people tend to prefer the target over the competitor. This switch in preference is considered irrational because it violates the principle of regularity, according to which the preference for an alternative cannot be increased by adding a new alternative to the choice set [43]. Participants in such experiments are not expected to choose the decoy as it is a dominated alternative.

\subsection{The attraction effect in visualizations}

The psychology literature had suggested that the attraction effect would disappear if more than three alternatives are added [7]. Nevertheless, work in visualization showed that the presence of multiple dominated datapoints in a scatterplot visualization does increase the attractiveness of other datapoints [22]. We summarize the experimental design and the main findings of that work, as we adopt a similar design.

\subsubsection{Experimental Design and Procedure}

Each participant was given 20 different choice tasks like the two seen in Fig. 3. For each task, the stimulus consisted of a scatterplot where the different options are lottery tickets. Each ticket has a probability of winning, and a prize (the amount that can be won). The options consisted of two optimal choices (one target and one competitor), and several decoys and distractors ${ }^{1}$. The relative positions of these different elements were counter-balanced among participants.

The participants, recruited on a crowdsourcing platform, first completed a tutorial and tests on probabilities and scatterplots. They were also informed that their remuneration depends on their performance.

\subsubsection{Findings}

As we see in Fig. 3, the points $\mathrm{A}$ and $\mathrm{C}$ are formally uncomparable: $\mathrm{A}$ is better on the horizontal dimension and $\mathrm{C}$ is better on the vertical dimension. Dimara et al. [22] observed that when A was the target, having multiple dominated points close to it (Fig. 3-left), people were more likely to choose A than C. On the other hand (Fig. 3-right), when dominated decoys were closer to C (target) people were more likely to select $\mathrm{C}$ over $\mathrm{A}$, even though the absolute values of $\mathrm{A}$ and $\mathrm{C}$ are identical in both cases. The reason for this inconsistency is the presence of the asymmetrically dominated datapoints (decoys).

\footnotetext{
${ }^{1}$ Distractors are irrelevant options that play neither the role of target, of competitor, or of decoy. In this case, distractors are the dominated alternatives that appear both in AC and CA choice tasks [22]
} 


\subsection{Is the attraction effect truly irrational?}

The attraction effect seems to be a robust cognitive bias that has been observed in many different contexts besides visualized data, such as when people buy commercial products [82], choose a meal in a menu [37], choose an employee to hire [41], and vote [39,67]. It has also been observed in animals like hummingbirds [5], and even brainless amoebae [55] when selecting their food.

However, as explained by Dimara et al. [22], in the case of visualizations part of the effect may also have perceptual origins. For example, regions with many decoys could be considered visually more salient than the isolated competitor. This does not invalidate the existence of the effect but it may provide additional explanation of why it occurs. Also, there are cases where decoys provide useful information that people can take into account in their decision. For example, a house in a region with many decoys may represent a larger market and therefore can be a more profitable investment [22]. In the example of the Bets experiment, though, this did not seem to be the case. Participants were asked after the study to interpret the presence of decoy lottery tickets but they did not appear to provide reasonable justifications, nor to be aware of their preference of the target ticket [22].

Generally, the concept of cognitive bias is controversial within the field of decision making [24]. Some researchers argue that cognitive biases illustrate irrationality [52], and others argue that some strategies that lead to biases can be effective in complex problems in the real world [33]. Therefore, InfoVis researchers are encouraged to verify if an erroneous response to a visualization task truly reflects irrationality, rather than a strategy based on alternative interpretation of the task [24].

\subsection{Alleviating the attraction effect with visualizations}

The attraction effect suggests that any decision involving a set of points that belongs to the Pareto front is influenced by the dominated datapoints below it (Fig. 2). When using a scatterplot to make such decisions, even if the data are appropriately visualized and fully understood, user decisions may still be affected by the irrelevant datapoints on the chart.

The simplest way to alleviate the attraction effect from a visualization would be to hide all dominated options (Fig. 2 A). However, hiding the points assumes that the system has full knowledge of the users decision criteria, which may not be the case in practice. Moreover, inferior options can help understand dataset trends, and may provide useful context when making decisions [22]. We therefore consider alternative methods for bias mitigation.

We test two methods that change the environment of the decision maker (Sect. 2.3) in an attempt to mitigate the bias. We suggest two approaches with which visualizations can reframe a decision problem:

1. Information presentation. A visualization system can alter the way information is presented to the user (e.g., it can change the visual encodings).

2. Information management A visualization system can alter the way the user manipulates the information (e.g., it can enrich the interactions provided).

The first experiment presented in this paper, called "PARETO", attempts to mitigate the attraction effect by following the information presentation approach. The second experiment, called "DELETION", follows the information management approach. Both experimental materials are available at http://www.aviz.fr/deletion.

\section{PARETO EXPERIMENT: THE VISUAL APPROACH}

In the PARETO experiment, we investigated whether by altering the visual representation of the data we can help users to be less affected by the attraction effect. We tested two conditions: baseline vs. pareto.

- The baseline condition uses exactly the same visual representation of the scatterplots in [22] as we see in the black-only plot in Fig. 3.

- The pareto condition only differs in indicating the Pareto front of non-dominated datapoints as we see in Fig. 4.

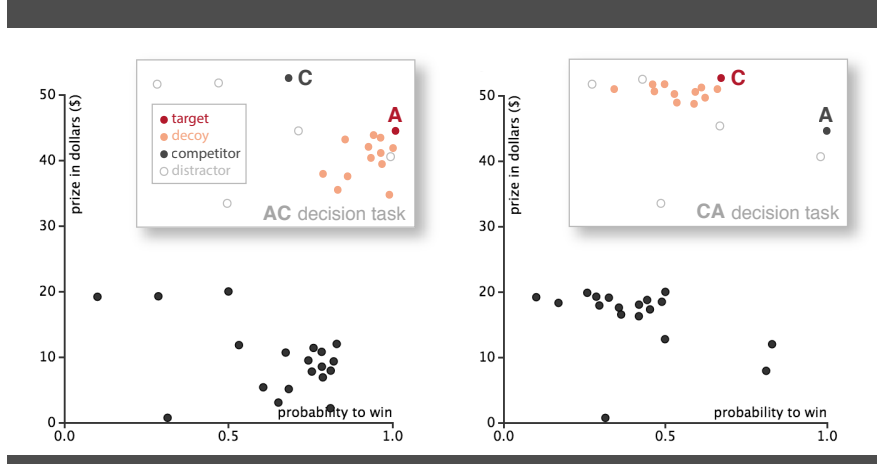

Fig. 3. Illustration of the attraction effect in scatterplot visualizations (the Bets experiment [22]). The position of the decoys affects the attractiveness of the target $\mathrm{A}$ for the left plot and $\mathrm{C}$ for the right plot. The letters $\mathrm{A}$ and $\mathrm{C}$, and coloring in the inlets are for illustration only, the actual stimuli were the scatterplots at the bottom.

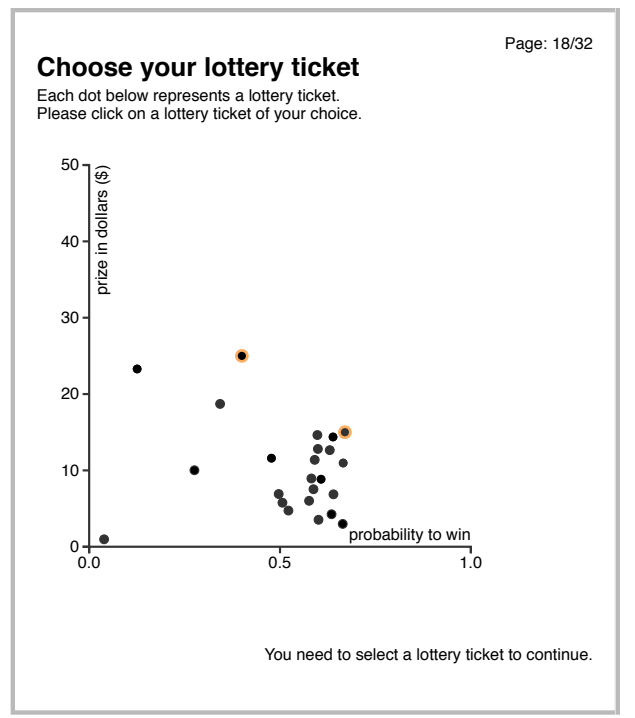

Fig. 4. Stimulus of the PARETO experiment. Non-dominated datapoints are indicated with a colored outline.

\subsection{Experiment rationale}

We changed the visual representation of the data to help users focus on the formally uncomparable (non-dominated) choices. An attraction effect choice task is divided into two subtasks [18]: the decision maker is expected to first recognize the dominance relationship among the different choices, consequently rejecting the $\operatorname{decoy}(\mathrm{s})$; and then to choose between the target and competitor. It is suggested [22] that the dominance recognition process (first step) causes the bias. It is thus possible that by indicating the non-dominated options, users will not attempt an effort-full dominance recognition task, avoiding the bias.

\subsection{Participants}

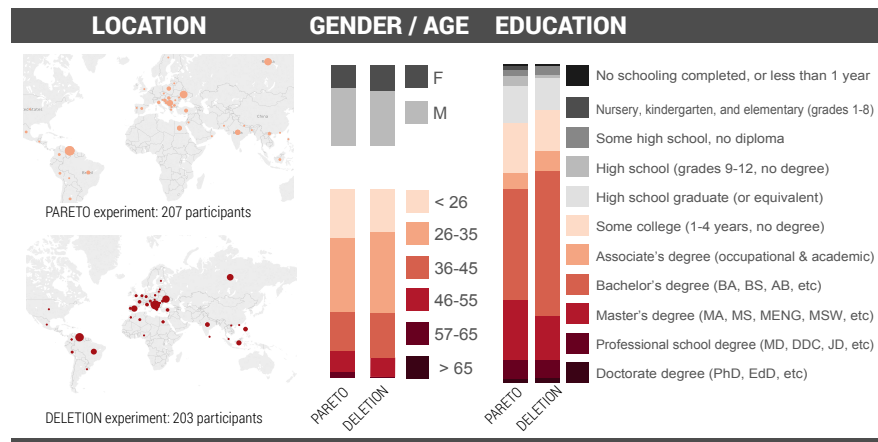

Fig. 5. Self-reported participant demographics in both experiments 
207 crowdsourcing participants submitted valid responses (107 for the pareto and 100 for the baseline condition ${ }^{2}$ ). Participants tended to be educated young men. Fig. 5 summarizes their self-reported demographics (map and bar charts). As in [22], they were paid $\$ 0.20$ with a bonus of $\$ 0.10$ and an extra expected lottery bonus of $\$ 0.50^{3}$. The actual average bonus payout $(\$ 0.48)$ was close to the expected bonus. Participants rated their payment satisfaction with 4.3 out of 5 stars on average (this rating was done before they receive their extra bonus payment). The task portion of the experiment lasted on average 8' 41 " for the baseline and 2' 14" for the pareto condition.

\subsection{Experimental design}

\subsubsection{Stimuli and Task}

The stimulus of the baseline condition was identical to the original study [22]: scatterplots showing the different lottery tickets options (Fig. 3). In the pareto condition, the black dots of the Pareto front were surrounded by an orange outline (Fig. 4) ${ }^{4}$. In both conditions participants had to select a ticket that they felt maximized their benefit.

\subsubsection{Procedure}

As in the original Bets experiment [22], we ran the study on a crowdsourcing platform. The experiment procedure started with a tutorial and a test on probabilities and scatterplots. As in [22], to better approximate a real-life decision, we informed participants that a computer will run the lottery, and for every winning ticket they picked, they will be paid a bonus proportional to the tickets prize. Then participants performed 20 choice tasks of lottery tickets presented in a scatterplot where they could select the lottery ticket of their choice.

Participants in the pareto condition got additional information before their first trial, explaining that the highlighted (orange outline) choices are optimal and thus indicate all possible correct choices of their previous test. We clarified again that these choices do not have the same probability or prize but that there is no wrong answer among them and their choice depends on their preferences.

For each trial, participants selected a lottery ticket by clicking on the corresponding point, and validated their answer by pushing a button.

\subsubsection{Design}

The PARETO experiment had one between-subjects factor the visual representation (baseline vs. pareto ). As in [22], the positions of the target, competitor and decoys were counter-balanced within participants. In total, each participant completed 20 trials (10 pairs of matched tasks like the $\mathrm{AC}$ and $\mathrm{CA}$ in Fig. 3).

\subsubsection{Measure: the attraction score}

To measure the attraction effect, we used the same attraction score formula as Wedell [90] (and [22]), which was calculated on a perparticipant basis. Each of the 20 decision tasks was assigned a score of 1 when the ticket with highest probability was chosen, of 0 when the ticket with highest prize was chosen, and a score of 0.5 when a dominated ticket was chosen. We averaged all scores for the 10 decision tasks where the decoys were on probability $\left(S_{\text {prob }}\right)$ and for the 10 tasks where the decoys were on prize $\left(S_{\text {prize }}\right)$. The difference $S=S_{\text {prob }}-S_{\text {prize }}$ is the attraction score. A participant not subject to the attraction effect should exhibit the same preference for high probability irrespective of the position of the decoys (contributing to both $S_{\text {prob }}$ and $S_{\text {prize }}$ ), thus her attraction score should be close to zero (for more details see [22]).

2 The planned size was 100 per condition (as slightly larger from the original study [22]), but the exact number is hard to precisely control in on-line platforms.

${ }^{3}$ After the experiment, we run an actual lottery to compute the bonus by i) running Bernoulli random draws to determine the winning status of each chosen ticket, ii) summing up the prizes of winning tickets, and iii) multiplying by a 0.0025 conversion rate from the scatterplot dollars.

${ }^{4}$ Another solution to indicate the Pareto is to connect the non-dominated options with a line. Since here we only had two non-dominated options, we chose to simply highlight them with a colored outline, rather than introducing an additional line encoding that needs to be explained. When more than two Pareto points are involved, both solutions should probably be considered.

\subsubsection{Hypothesis}

The research hypothesis was:

Hr1 Indicating the non-dominated datapoints in a scatterplot reduces the attraction effect.

This translates into the following statistical hypothesis:

H1 The mean attraction score of the pareto condition will be smaller than the mean attraction score of the baseline condition.

\subsection{Planned Analysis Results}

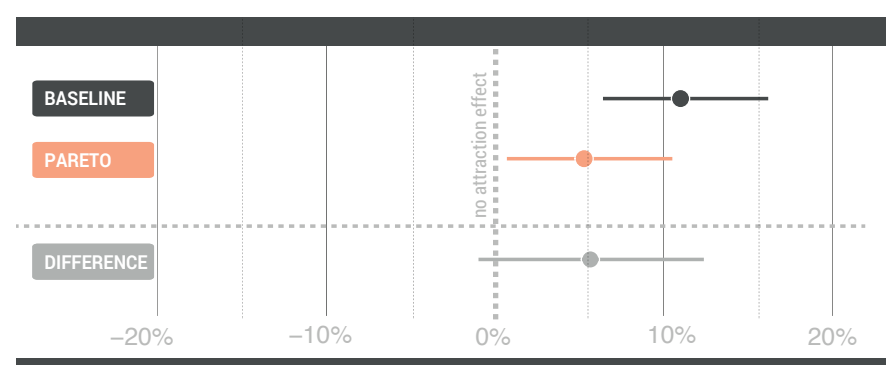

Fig. 6. PARETO experiment: point estimates and $95 \%$ confidence intervals for the attraction effects in baseline and pareto conditions.

We analyze, report and interpret all our inferential statistics using interval estimation [25]. All analyses reported in this section were planned before data was collected.

For the baseline condition, the total number of trials were 2000 (100 participants X 20 decision tasks). For the pareto condition, we recorded a total of 2140 (107 participants X 20 decision tasks).

Participants sometimes chose a dominated alternative instead of the target or competitor. In the PARETO experiment, this was true for $2.4 \%$ choice pairs (e.g., task pairs AC and CA in Fig. 3) for baseline and for $5.33 \%$ for pareto. This percentage was $3.3 \%$ in the original paper [22].

For the baseline condition, the mean attraction score $S_{\text {baseline was }}$ $11 \%$, with a $95 \%$ bootstrap confidence interval of $[6 \%, 16 \%]$ (see Fig. 6). Like [22], there is still solid evidence that participants were subject to the attraction effect.

For the pareto condition, where participants saw the dominant datapoints highlighted, the mean attraction score $S_{\text {pareto }}$ was $5 \%$, with a $95 \%$ bootstrap confidence interval of $[0.7 \%, 11 \%]$ (see Fig. 6 ). The range of plausible mean values of the effect indicates that the effect is relatively smaller.

The difference $D=S_{\text {baseline }}-S_{\text {pareto }}$ in means between the two conditions was $6 \%$, with a $95 \%$ bootstrap confidence interval of $[-1 \%$, $12 \%$ ]. Thus, there is some evidence that the hypothesis $\mathbf{H 1}$ is true.

\subsection{Discussion}

We found suggestive evidence that highlighting the Pareto front helped participants to make faster decision and weaken the attraction bias, i.e., it reduced somewhat the influence of decoys. Focusing on the Pareto front may have facilitated a 'fast' mode of reasoning, but it did not eliminate the bias, though different highlighting techniques (e.g., using a stronger visual cue, such as a line) should be tested. Even if such techniques were more successful in mitigating the bias, any form of highlighting optimal datapoints would have other limitations. First, there may not always be a formal definition of optimality that a visualization system could use to highlight points. There are also other biases were highlighting the pareto front is not effective, for example the compromise effect [81], where people chose more 'average' values from within the Pareto front; or the phantom effect [73], where unavailable alternatives influence choices within the Pareto set. 


\section{Deletion rationale}

We previously investigated a visual solution to alleviate a decision bias. In this section, we search for ways to improve the interactions involved in the reasoning process by reviewing recommended strategies from decision theory. We first describe the two main families of these strategies, named compensatory and non-compensatory and explain our choice for the second. We then explain our focus on the noncompensatory strategy named "elimination by aspects" (EBA). We finally discuss how we can implement EBA in a visualization tool.

\subsection{Compensatory strategies}

Multi-attribute choice models suggest various strategies to support consistency in human decision making [74]. One family of strategies is called compensatory, in which the superior value of one attribute compensates for the inferior value of another attribute [74]. One compensatory strategy is the "weighted additive" (WADD), in which the decision maker weights all attributes by importance and chooses the one with the highest weighted sum [72]. Many visualization tools [12,35, 70] support the WADD by allowing users to combine attributes into a single aggregate score. They initially show the data as a tabular visualization where columns can be resized to express attribute importance. The entire visualization can then be collapsed into a stacked bar chart and sorted. Many other compensatory strategies exist: the "equal weight" (EQW) which sums alternatives without considering weights of importance [72], or the "additive difference" (ADDIF), which evaluates first each attribute across alternatives, and then weights and sums only their differences [74].

\subsection{Non-compensatory strategies}

Models that do not consider such tradeoffs are called non-compensatory [74]. Decision makers can declare a threashold for an attribute, and drop alternatives with bad values, even if they has high values for the other attributes [94]. One of the oldest non-compensatory strategies is the "satisficing" (SAT) [80], in which the decision maker first assigns thresholds, evaluates the alternatives by order of appearance, and chooses the first that satisfies these thresholds. If no such alternative exists, the decision maker relaxes the thresholds and repeats the process, or chooses a random alternative [72]. Another non-compensatory strategy is the "lexicographic" (LEX) one, where the decision maker identifies the most important attribute, and then chooses the alternative with the best value for this attribute. If more than one alternatives exist, they identify the second most important attribute and repeats [74]. Finally, another non-compensatory strategy is the "elimination by aspects" (EBA) proposed by Tversky in 1972 [86] that is very similar to the LEX, except that instead of choosing the best alternative for the most important attribute, the decision maker rejects all alternatives that do not satisfy a given threshold and repeats until only one alternative is left [74].

\subsection{Choosing a strategy to mitigate the attraction effect}

Compensatory strategies require a decision maker to consider attribute trade-offs, which people find uncomfortable to deal with [72]. These negative emotions may be driven by the difficulty of calculating how much of one attribute to give up in exchange for another [38], e.g., calculating how much of prize to give up in exchange for the probability to win. In contrast, when a decoy is present near the target, these negative emotions decrease [38], presumably because people can now focus on the decoy vs. the target, a non-compensatory strategy that allows them to avoid considering tradeoffs. But the non-compensatory strategy of focusing only on the decoy and target may be a cause of the attraction effect.

Because people prefer non-compensatory strategies, we therefore chose to test one taken from the decision making literature, ELIMINATION BY ASPECTS (EBA) [86]. This strategy should allow the decision maker to minimize explicit consideration of trade-offs, in a way that avoids the attraction effect, by promoting a slower, more deliberate form of local decision-making. In EBA, the decision maker first rejects all the alternatives that do not satisfy her choice criteria to end up with the alternative of her choice. Similarly, as we explained in Sect. 3.4, a typical attraction effect choice task is divided into two subtasks: the decision maker is expected to first recognize the dominant points by rejecting the $\operatorname{decoy}(\mathrm{s})$, and, second to choose between the two trade-off choices [18]. Since the first step is often claimed to cause the bias, in DELETION experiment, we attempt to differentiate these two tasks (dominance recognition and choice) with interaction, so that the dominance recognition task does not affect the final decision.

\subsection{Implementing the elimination by aspects (EBA)}

In the EBA, unwanted alternatives are removed from a dataset. We explore how to implement this strategy in a visualization tool.

\subsubsection{Deletion in visualization}

As deletion we refer to the task of removing one or more data cases from a visualized dataset. Deletion is a low-level analytic task [15] that is usually omitted from most visualization taxonomies [1,78, 85,91,95]. We consider two ways to delete data from a visualization tool:

- Filter-based deletion: A common task in visualization systems is to perform a filter-based deletion by removing data cases "based on their values concerning specific attributes" [63]. Filter-based deletion of data cases is a common interaction during visual analysis tasks. Consider a dataset with two data points A and B with values $X_{A}>X_{B}$ for a given attribute $\mathrm{X}$. If a user wants to remove $B$ as an outlier because of its high $X$ value (e.g., to reduce clutter), she will also want to remove outlier A. This is a common filter-based deletion based on a value threshold.

- Local deletion: As local deletion we define the task of removing data cases that have been explicitly identified by the user. A local deletion of data cases is a less common interaction. In the example above, deleting only the outlier B without a link to other dataset features would likely be a source of confusion.

Although filter-based deletion and local deletion sound similar, they are not equivalent: For instance, a visualization user who wants affordable houses, can remove data cases based on their price (filter-based deletion). If she does not like the photo of a house (or due to other criteria not included in the dataset) she can also remove the specific house data case (local deletion). The filter-based deletion reflects a rule in which all data-cases that meet it are removed (e.g., all expensive houses). In local deletion, the data cases with similar or even identical house photos will not be removed as the system is not aware of the removal criteria. Most decision-support visualizations [23] allow filterbased deletion but do not support local deletion features. For example, in LineUp, the user can not directly remove a row from the table, but she can remove rows by specifying attribute filters [35].

\subsubsection{Deletion for mitigating the attraction effect}

Both filter-based and local deletion might help reduce the attraction effect. The dominated datapoints could be removed once the user indicates a threshold of their attributes based on the target and the competitor. However, our goal is to propose an interaction that can later be used to support real-world decision tasks and help decision makers to remove irrelevant information that cannot be summarized by thresholds for dataset attributes.

Local deletion can be particularly useful in decision making. First, it is likely that a decision maker will weigh information not included in the dataset, e.g., to rule out failed solutions that she tried in the past. Moreover, even if the decision maker wants to make a decision based on information that exists in the dataset, she may not know a precise rule for the criteria. For example, the house buyer may delete a house she finds too expensive for having only one bathroom and no other particular appealing attribute. This does not imply that she wants all expensive houses with one bathroom to be removed (e.g., she could compromise with a house with other room surplus and appealing big garden). Choice preferences can evolve during exploration and are often formed progressively based on the availability of the alternatives.

In the next section, we test whether decision makers who can locally "clean up" the decision space from irrelevant information are less vulnerable to the attraction effect. 


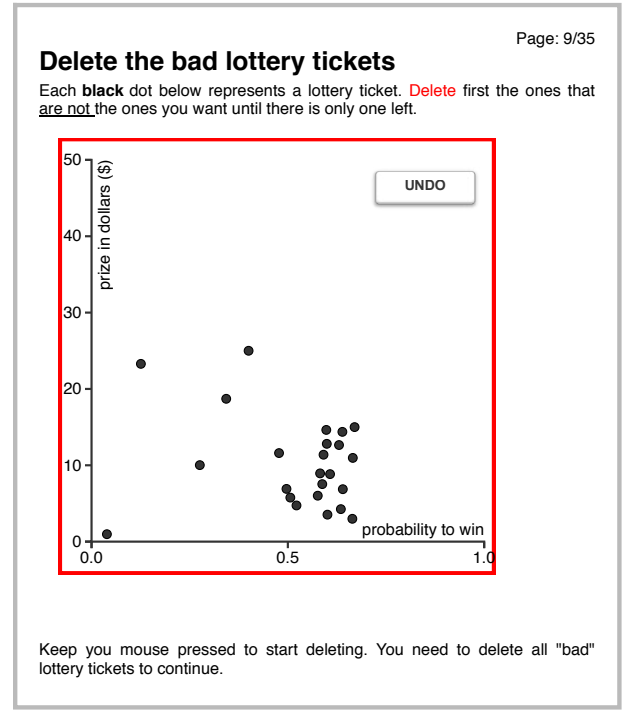

Fig. 7. Stimulus of the DELETION experiment. While the mouse was pressed, a red outline indicated that the mouse will be deleting datapoints. Participants deleted all datapoints but one - their final choice.

\section{DELETION EXPERIMENT: THE INTERACTION APPROACH}

In the DELETION experiment, we investigated whether altering the way visualization users interact with the data can be an effective debiasing approach. In particular, we examined whether enforcing the elimination by aspects decision strategy will make participants less vulnerable to the attraction effect. We tested two conditions: baseline vs. deletion.

- The baseline condition uses exactly the same interaction as in [22], where participants directly selected the alternative of their choice.

- The deletion condition uses an interaction where participants first deleted all the unwanted alternatives to end up with the alternative of their choice as in Fig. 7.

\subsection{Experiment rationale}

Our method consists of an interaction of local deletions to encourage users alter their decision strategy. From decision theory (Sect. 5 ), we adopt a well-known strategy of "elimination by aspects" (EBA) in which the decision maker discards undesired alternatives in early stages of the decision process. In visualizations, this strategy can be translated to an interaction that allows users to directly delete the visualized data.

\subsection{Participants}

203 crowdsourcing participants submitted valid responses (102 for the baseline and 101 for the deletion condition ${ }^{5}$. Participants had similar self-reported demographics to the PARETO experiment (see Fig. 5) and received the same payment (Sect. 4.2). The actual average bonus payout was $\$ 0.50$. The task portion of the experiment lasted on average 6'28" (baseline condition) and 14'07' (deletion condition). Participants rated their payment with 3.8 out of 5 stars before receiving their bonus.

\subsection{Experimental design}

\subsubsection{Stimuli and Task}

In both conditions the task was the same as in the PARETO experiment: twenty choice tasks of lottery tickets each defined by a probability to win, and a prize (amount that can be won). Each choice task consisted, again, of a target, a competitor, decoys and distractors. The stimuli of both baseline and deletion conditions were identical: scatterplots with black dots representing lottery tickets (seen in Fig. 3).

However, the procedure that a participant had to follow in order to complete the task differed. In the baseline condition, participants directly selected their choice as in the PARETO experiment by clicking

\footnotetext{
${ }^{5}$ The planned size was 200, like in the PARETO experiment, which was again hard to precisely control in the online platform.
}

on it, and confirming their choice on a button at the bottom of the page. To undo a selection, the user could click again anywhere else apart from the confirmation button. Dots were highlighted when hovered. Hovering a dot also displayed horizontal and vertical projection lines, and the dot's $X$ and $Y$ values were overlaid on the axes. ${ }^{6}$

In the deletion condition, participants had to first delete all the unwanted alternatives to end up with the alternative of their choice. While the mouse was pressed, a red outline was displayed around the scatterplot to indicate that the user is on deletion mode (seen in Fig. 7). During deletion mode, dragging over a dot would remove it. The user could also delete dots by instantly clicking on them. The deletion area was substantially smaller than the size of the dot. This made the deletion more tedious, but it was necessary to prevent errors since some decoys were very close to the target and to each other. To undo a deletion, an "undo" button was offered to recover the deleted dots. The hovering feature was the same as in the baseline condition.

\subsubsection{Procedure}

In the baseline condition, participants followed exactly the same procedure as in the baseline of the PARETO experiment clicking on the lottery ticket of their choice (Sect. 4.3.2).

Each participant of the deletion condition followed the same procedure as in the baseline and saw the same trials, with two differences. First, before their main choice tasks, in order to prevent accidental deletions, participants went through a deletion training and pre-test. They were given the following piece of instructions "During the tutorial", you clicked on a dot to select it. You will not do this anymore. First, you have to delete the dots you do not want. The last dot you will leave will be the one you select.". Then, red and black dots appeared in the screen, instructing them to delete the red and leave only the black dots in order to proceed to the experiment. The position of the dots was carefully arranged so as not to prime users by showing a pattern similar to the attraction effect, and to also require a precise deletion of a red dot being very close to a black one. If the participant deleted by mistake a red dot, she was forced to press the undo button to redo the task from scratch. The second difference was that to choose a lottery ticket in the scatterplot, participants had to delete first all unwanted alternatives in order to select the last one left as we described in Sect. 6.3.1.

\subsubsection{Design}

The DELETION experiment had one between-subjects factor the interaction technique (selection or deletion). As in the PARETO experiment, the positions of the target, competitor and decoys were counter-balanced within participants. In total, each participant completed 20 trials (10 pairs of matched tasks like the AC and CA in Fig. 3).

\subsubsection{Hypothesis}

The research hypothesis was:

Hr2 Following the elimination by aspects decision strategy while interacting with a scatterplot, reduces the attraction effect.

This translates into the following statistical hypothesis:

H2 The mean attraction score of the deletion condition will be smaller than the mean attraction score of the baseline condition.

\subsection{Planned analysis results}

For the baseline condition, the total number of trials were 2040 (102 participants X 20 decision tasks). For the deletion condition, we recorded a total of 2020 (101 participants X 20 decision tasks).

We first report how often participants chose a dominated alternative instead of a target or competitor. For the original study [22] this value was $3.3 \%$ of the choice pairs, and in the DELETION experiment it was $3.3 \%$ for baseline, and $1.39 \%$. for deletion. Thus error trials (choice of decoys) were as low or lower than the original experiment.

\footnotetext{
${ }^{6}$ We note that this hovering functionality also existed in the original experiment and in the PARETO experiment.

${ }^{7}$ The tutorial refers to the test of probabilities and scatterplots.
} 


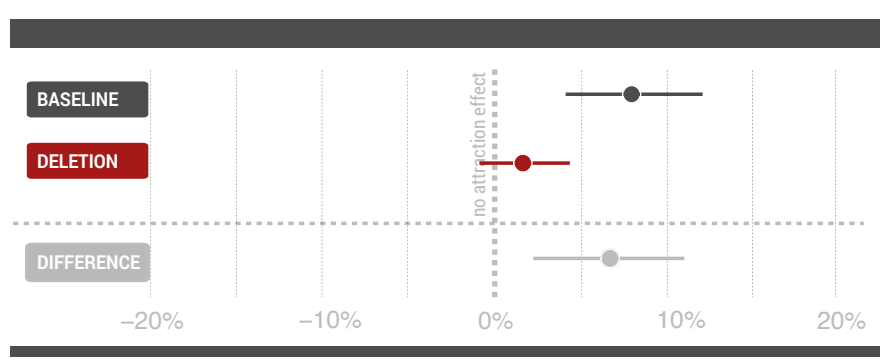

Fig. 8. Point estimates and $95 \%$ confidence intervals for the attraction effects in baseline and deletion conditions.

The attraction score, as explained in the PARETO experiment, was based on the difference in choices made when the decoys were on prize and the choices made when the decoys were on probability. A participant whose preference for high probability or prize is independent from the position of the decoys should have attraction score close to zero.

For the baseline condition, where participants directly clicked on the ticket of their choice, the mean attraction score $S_{\text {baseline }}$ was $8 \%$, with a $95 \%$ bootstrap confidence interval of $[4 \%, 12 \%]$ (see Fig. 8). Like in the previous Bets experiment, there is still strong evidence that participants were subject to the attraction effect.

For the deletion condition, where participants deleted first the unwanted tickets, the mean attraction score $S_{\text {deletion }}$ was $1.3 \%$, with a $95 \%$ bootstrap confidence interval of $[-1.3 \%, 4 \%]$ (see Fig. 8). The range of plausible mean values of the effect indicates that either participants were not subject to an attraction effect or that the effect is small.

The difference $D=S_{\text {baseline }}-S_{\text {deletion }}$ in means between the two conditions was $7 \%$, with a $95 \%$ bootstrap confidence interval of $[2 \%$, $11 \%$ ]. Thus, there is strong evidence that the hypothesis $\mathbf{H 2}$ is true.

\subsection{Exploratory analysis of participant behavior}

We performed an exploratory analysis of the interactions of the 101 participants of the deletion condition, in order to better understand if there were consistent patterns or strategies in how participants used this novel way of selecting points that might be relevant for future work We anticipated that most participants would keep the two best options until the end of the trial, and would thus be less affected by the presence of decoys when making a decision. This behavior could potentially explain the alleviation of the attraction effect.

\subsubsection{When did participants delete targets and competitors?}

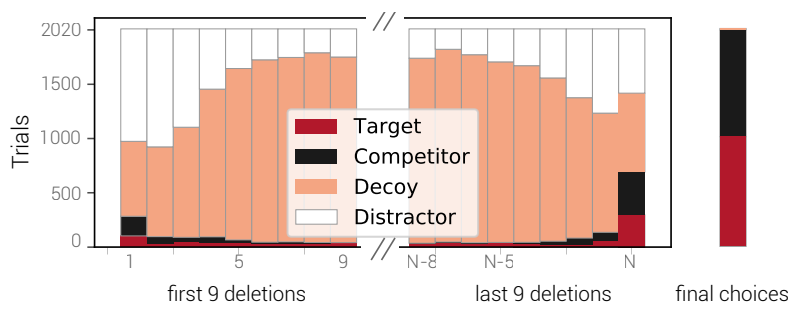

Fig. 9. The number of targets, competitors, decoys and distractors for the first 9 and last 9 deletions of the $100 \times 20=2000$ trials. $N$ is the number of deletions (click on a dot). The right bar shows the final total selections. The selection was implicit by deleting (click on) all unwanted dots.

Fig. 9 summarizes when participants deleted the items (target, competitor, decoy and distractor) within trials. The additional bar on the right shows the final selection (implicit selection via deleting all other datapoints). In $35 \%$ of the trials, the last point to be deleted was either the target or the competitor (N-bar). Conversely, in $65 \%$ of the trials, one or several decoys still remained on the screen when participants deleted the target or the competitor. A closer look at the data reveals that for $49 \%$ of trials, the target or the competitor are removed in the $20 \%$ last deletions (corresponding to 3-5 deletions depending on number of items in the trial). This indicates that while participants did not wait for the very last deletion to remove the target or the competitor, they still had a tendency to remove them late in the trial (i.e., in roughly half the trials $80 \%$ of the decoys had already been removed). Surprisingly, we also observed that in some trials (30\%), targets and competitors were deleted early (first $20 \%$ deletions). This might suggest that in these trials participants first made a decision and then mechanically deleted datapoints, i.e., did not benefit from the technique as all decoys were still visible when they made their decision.

Observation 1. In roughly half of the trials target and competitor were kept until the last $20 \%$ of a trial.

\subsubsection{How did participants use the deletion?}

We anticipated that participants would remove decoys by moving closer to the target, i.e., keeping the best options last. To investigate participant spatial strategies when deleting decoys, we displayed the distance of the deleted decoys from the target as a function of trial progress $(\%)$ for each participant. Two annotators independently observed the data and both identified three strategies (DECREASE; INCREASE; OTHER) illustrated in Fig. 10. They then independently assigned each participant to one strategy with an agreement of 0.75 (Cohen's Kappa) before resolving disagreements. As anticipated, the first strategy, DECREASE (30 occurrences), illustrated with the first participant consists of deleting decoys by moving closer to the target. In contrast, the second strategy, INCREASE (22 occurrences), is illustrated with the second participant and consists of deleting decoys by moving away from the target. In these cases users may have tried to reduce the distance between two consecutive decoys in order to decrease the cumulative selection time [57]. Nevertheless, for the last group of participants, OTHER (49 occurrences), illustrated with the third participant, no tendency is visible. This OTHER strategy is less optimal from a motor control perspective. In summary, there is no strategy that clearly pops out and the anticipated one (DECREASE) is used by $30 \%$ of the participants. The data also showed that the same participant does not seem to follow the same strategy from one trial to another. While it is possible that optimising motor control, or other interaction constraints (e.g., handedness), could influence deletion strategies, the mix of strategies observed in our experiment is not enough to draw such a conclusion. Further research is needed to verify this. While this analysis could be informative, the deletion strategy that participants followed is not necessarily equivalent to their actual decision strategy. That is, we cannot speculate which decision strategy people had in mind while mechanically deleting datapoints.

Observation 2. No consistent spatial pattern among participants for removing decoys.

We also investigated whether the chosen strategy influences when target and competitor are deleted and ultimately their final selection. Results suggest that participants removed target and competitor later in the trial (i.e. last 20\% deletions) with DECREASE (65\%) than OTHER $(46 \%)$ and INCREASE (36\%). However, the final impact of strategy on target and competitor selection seems not to indicate any difference: DECREASE: $51 \%$ vs. $49 \%$; DECREASE: $49 \%$ vs. $51 \%$; OTHER: $50 \%$ vs. $50 \%$. These numbers are only given as an indication due to small sample size. Future work is necessary to validate these assumptions.

\subsubsection{Was deletion more error prone?}

One possible concern is that some participants accidentally deleted the target or the competitor. Considering (i) the very low error rate of the deletion condition (1.39\%, lower than the baseline condition); (ii) participants' tendency to often delete the target/competitor among last items; (iii) the small area of the deletion brush that makes multiple point deletion hard; (iv) the use of the UNDO button (mean=0.2 actions per participant; $s d=0.8 ; \max =7$ ), indicating that, even though they did occasionally make accidental deletions, at least in some cases they were aware of it and did correct them, we believe that accidental deletions of target or competitor were not very common. Nevertheless, another type of experimental design that captures participants' intent is needed to conclusively determine if the technique promotes accidental deletions. 


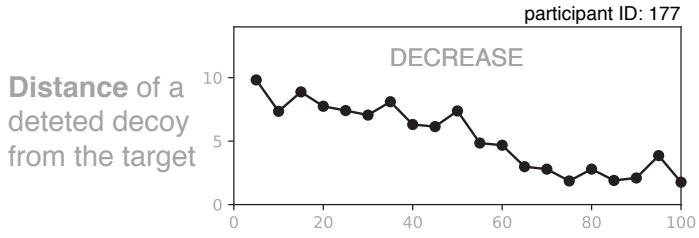

trial progress $(\%)$

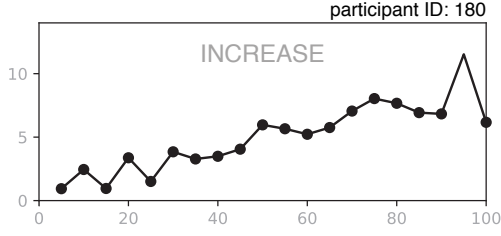

trial progress $(\%)$

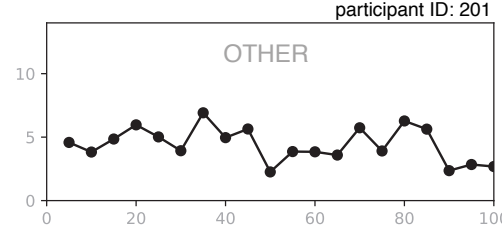

trial progress $(\%)$

Fig. 10. Three spatial deletion strategies show the distance of a deleted decoy from the target as a function of the trial progress. The 1st participant tends to start deleting decoys far from the target. The 2 nd participant starts deleting decoys close to the target. No visible pattern for the 3 rd.

\subsection{Discussion}

The DELETION experiment confirms that following the elimination by aspects (EBA) decision strategy while interacting with a scatterplot reduces the attraction effect. It showed that it is possible to provide interactions that simulate well-identified decision strategies to help users make more rational decisions. This finding is especially interesting as most debiasing techniques usually require extensive training (formal courses, tutorials or video game simulations [16], computation aid, etc.) with limited results.

Local deletion led to more rational decisions (in the sense of being consistent with axioms of rational choice theory), and we speculate below on how future work might disentangle the potential reasons why.

\subsubsection{Time and effort}

In the DELETION experiment participants were asked to make a good decision rather than a fast one. Even though deleting alternatives seems to yield more rational decisions, participants spent twice as much time as those in the baseline condition. There are at least two potential reasons for the longer response times:

- Motor. The deletion condition relied on a small brush size, to avoid accidental deletions when the decoys were too close to the target, as explained in Sect. 6.3.1. The option of a larger deletion area could improve the overall task time, but it would still be impossible to compete with a direct click on a single dot or it could also lead to more undo operations.

- Cognition. There is an inevitable cost of enforcing a structured decision making strategy because decision makers need to explicitly iterate over almost all alternatives. On the other hand, the baseline condition did not enforce any strategy. So, it is possible that participants followed a strategy like (EBA) or select the first option that satisfies their needs (SAT).

While deletion was more time consuming, it is possible that the additional time, per se, had a positive effect on their reasoning, beyond the effects of deleting irrelevant values. The additional time could have allowed them to consider their choices more thoroughly. Future work might make the baseline condition equally time consuming, for example, by freezing the screen for a few minutes, to test the contribution of additional decision time, per se. On the other hand, extended wait time does not guarantee extra cognitive effort—a delay might merely frustrate or bore the user, and cause her to switch to another task

\subsubsection{Deletion benefits and deployment}

There are more plausible explanations as to why the deletion condition led to more rational decisions. It is possible that "cleaning up" the decision space from irrelevant information helped participants to focus on the important information (in half the trials $80 \%$ of the decoys were removed when the decision was made). The process of deleting decoys may have helped them to consciously strengthen the idea that the deleted information is irrelevant or unimportant. Also, the decision strategy enforced by interactions in the deletion condition reduces the distress of comparing trade-offs between attributes, which may reduce reliance on heuristic comparisons to decoys, which in turn may reduce the attraction effect. Finally, the more tedious interaction may have enforced a more slow procedural process, going back an forth between delete actions and cognitive re-evaluation of alternatives to delete.
The purpose of enforcing point-by-point deletion was to study its effect on the attraction effect in a controlled experimental setting. How might this method be implemented in real a application? One clear strategy would be to provide flexible local deletion methods (e.g., a larger deletion area, or lasso tool) to save time. Of course, deletion tools should be optional, and not replace direct selection of the desired choice. Another consideration is how to guide users to optimize their decision process by deleting unnecessary information, while maintaining information that is important.

In the DELETION experiment we asked users to perform an otherwise easy task (only two obvious good alternatives) in a "tedious" way, which would not make sense in a real system. We generally think that wellstructured decision strategies like EBA would be practically useful for decision-support visualization systems that are meant to support more complex tasks with multiple alternatives and attributes. Such systems are encouraged to enrich their interaction palette to implement various decision strategies and help users to better manage and likely reduce the visual clutter. An example of using interactions to support a strategy is illustrated by systems such as Value Charts [6] which encourage the weighted additive strategy by allowing weight manipulations of importance and aggregation through stacked bar visualizations.

\section{ConClusion}

Debiasing is often associated with educating people with extensive training or video game simulations, typically with little success. We focused instead on the system, providing the first empirical evidence that interactive visualizations can be effectively used to mitigate cognitive biases. In our displays, the attraction effect stems from an inappropriate weighting of decoy objects, and a rational decision maker should only consider points within the Pareto front. We found that a non-interactive approach, the highlighted Pareto front, led to weak evidence for a drop in the attraction effect, presumably by helping the participant ignore the biasing information. Forcing the user to interact and progressively delete suboptimal points led to a strong drop, by explicitly removing the biasing information from the display.

Together, the PARETO and DELETION experiments suggest two methods that may help reduce biases in decision making within data visualizations: computational aids that highlight optimal decisions based on objective criteria, but more counterintuitively, a system where users can systematically delete information as they make comparative decisions at a more local level of analysis.

At first glance, removing information from a display appears to be an extreme solution, because the power of visualizations is that they allow us to process massive amounts of data at once - why lose information and context? Yet the attraction effect is an example of how this context can mislead. And visualizations already constantly omit information, with lower-dimensional views of complex datasets, parameter slides, and other focusing techniques. Why not allow users to remove information by hand, instead of only with automated processes? Visualizations are tools for information management, and this type of by-hand management might benefit a wider variety of visualizations.

\section{ACKNOWLEDGMENTS}

We thank P. Dragicevic, S. Haroz, and I. Avellino for their precious feedback. 


\section{REFERENCES}

[1] R. Amar, J. Eagan, and J. Stasko. Low-level components of analytic activity in information visualization. In Information Visualization, 2005. INFOVIS 2005. IEEE Symposium on, pp. 111-117. IEEE, 2005.

[2] E. B. Andrade. Excessive confidence in visually-based estimates. Organizational Behavior and Human Decision Processes, 116(2):252-261, 2011.

[3] H. R. Arkes. Costs and benefits of judgment errors: Implications for debiasing. Psychological bulletin, 110(3):486, 1991.

[4] A. K. Barbey and S. A. Sloman. Base-rate respect: From ecological rationality to dual processes. Behavioral and Brain Sciences, 30(03):241254, 2007.

[5] M. Bateson, S. D. Healy, and Hurly. Irrational choices in hummingbird foraging behaviour. Animal Behaviour, 63(3):587-596, Mar. 2002. doi: 10.1006/anbe.2001.1925

[6] J. Bautista and G. Carenini. An empirical evaluation of interactive visualizations for preferential choice. In Proc. of the working conference on Advanced visual interfaces, pp. 207-214. ACM, 2008.

[7] J. R. Bettman, M. F. Luce, and J. W. Payne. Constructive consumer choice processes. J. of Consumer Research, 25(3):187-217, Dec. 1998.

[8] J. Boy, A. V. Pandey, J. Emerson, M. Satterthwaite, O. Nov, and E. Bertini Showing people behind data: Does anthropomorphizing visualizations elicit more empathy for human rights data? In ACM Conference on Human Factors in Computing Systems, pp. 5462-5474. ACM, 2017.

[9] C. L. Brown. do the right thing: diverging effects of accountability in a managerial context. Marketing Science, 18(3):230-246, 1999.

[10] M. R. Cadinu and M. Rothbart. Self-anchoring and differentiation processes in the minimal group setting. Journal of personality and social psychology, 70(4):661, 1996.

[11] C. F. Camerer, R. M. Hogarth, D. V. Budescu, and C. Eckel. The effects of financial incentives in experiments: A review and capital-labor-production framework. In Elicitation of Preferences, pp. 7-48. Springer, 1999.

[12] G. Carenini and J. Loyd. Valuecharts: analyzing linear models expressing preferences and evaluations. In Proc. of the working conference on Advanced visual interfaces, pp. 150-157. ACM, 2004.

[13] P. Cheng, K. Holyoak, and R. Nisbett. Pragmatic versus syntactic approaches to training deductive reasoning. Cognitive Psychology, 18:293328, 1986.

[14] I. Cho, R. Wesslen, A. Karduni, S. Santhanam, S. Shaikh, and W. Dou. The anchoring effect in decision-making with visual analytics. IEEE Transactions on Visualization and Computer Graphics, 2018.

[15] M. C. Chuah and S. F. Roth. On the semantics of interactive visualizations. In Information Visualization'96, Proc. of IEEE Symposium on, pp. 29-36. IEEE, 1996.

[16] B. A. Clegg, R. M. Martey, J. Stromer-Galley, K. Kenski, T. Saulnier, J. E. Folkestad, E. McLaren, A. Shaw, J. E. Lewis, J. D. Patterson, et al. Game-based training to mitigate three forms of cognitive bias. In Proc. of Interservice/Industry Training, Simulation and Education Conference (I/ITSEC), vol. 14180, pp. 1-12, 2014.

[17] M. Correll and M. Gleicher. Error bars considered harmful: Exploring alternate encodings for mean and error. Visualization and Computer Graphics, IEEE Transactions on, 20(12):2142-2151, 2014

[18] P. Crosetto and A. Gaudeul. Testing the strength and robustness of the attraction effect in consumer decision making. 2015.

[19] P. Croskerry, G. Singhal, and S. Mamede. Cognitive debiasing 1: origins of bias and theory of debiasing. BMJ quality \& safety, 22(Suppl 2):ii58-ii64, 2013.

[20] M. Cummings. Automation bias in intelligent time critical decision support systems. In AIAA 1st Intelligent Systems Technical Conference, p. 6313, 2004.

[21] R. M. Dawes, D. Faust, and P. E. Meehl. Clinical versus actuarial judgment. Science, 243(4899):1668-1674, 1989.

[22] E. Dimara, A. Bezerianos, and P. Dragicevic. The attraction effect in information visualization. IEEE Transactions on Visualization and Computer Graphics, 23(1):471-480, 2017.

[23] E. Dimara, A. Bezerianos, and P. Dragicevic. Conceptual and methodological issues in evaluating multidimensional visualizations for decision support. IEEE Transactions on Visualization and Computer Graphics, 2018.

[24] E. Dimara, S. Franconeri, C. Plaisant, A. Bezerianos, and P. Dragicevic A task-based taxonomy of cognitive biases for information visualization.

[25] P. Dragicevic. Fair statistical communication in HCI. In Modern Statistical
Methods for HCI, pp. 291-330. Springer, 2016

[26] N. E. Dunbar, M. L. Jensen, B. Miller, Adame, et al. Mitigating cognitive bias through the use of serious games: Effects of feedback. In International Conference on Persuasive Technology, pp. 92-105. Springer, 2014.

[27] B. Fischhoff. Debiasing/kahneman, d., slovic, p. and tversky, a. In D. Kahneman, P. Slovic, and A. Tversky, eds., Judgment Under Uncertainty: Heuristics and Biases. Cambridge University Press, 1982.

[28] B. Fischhoff, P. Slovic, and S. Lichtenstein. Knowing with certainty: The appropriateness of extreme confidence. Journal of Experimental Psychology: Human perception and performance, 3(4):552, 1977.

[29] R. J. Fisher. Social desirability bias and the validity of indirect questioning. Journal of consumer research, 20(2):303-315, 1993.

[30] G. T. Fong, D. H. Krantz, and R. E. Nisbett. The effects of statistical training on thinking about everyday problems. Cognitive psychology, 18(3):253-292, 1986.

[31] G. T. Fong and R. E. Nisbett. Immediate and delayed transfer of training effects in statistical reasoning. Journal of Experimental Psychology: General, 120(1):34, 1991

[32] E. R. Fyfe, N. M. McNeil, J. Y. Son, and R. L. Goldstone. Concreteness fading in mathematics and science instruction: A systematic review. Educational Psychology Review, 26(1):9-25, 2014.

[33] G. Gigerenzer and H. Brighton. Homo heuristicus: Why biased minds make better inferences. Topics in cognitive science, 1(1):107-143, 2009.

[34] M. L. Graber, S. Kissam, V. L. Payne, A. N. Meyer, A. Sorensen, N. Lenfestey, E. Tant, K. Henriksen, K. LaBresh, and H. Singh. Cognitive interventions to reduce diagnostic error: a narrative review. BMJ Quality \& Safety, pp. bmjqs-2011, 2012.

[35] S. Gratzl, A. Lex, N. Gehlenborg, H. Pfister, and M. Streit. Lineup: Visual analysis of multi-attribute rankings. IEEE transactions on visualization and computer graphics, 19(12):2277-2286, 2013.

[36] G. Grinstein, J. C. Sieg, S. Smith, and M. G. Williams. Visualization for knowledge discovery. International Journal of Intelligent Systems, 7(7):637-648, 1992.

[37] A. S. Hanks, D. R. Just, and B. Wansink. Trigger foods: The influence of irrelevant alternatives in school lunchrooms. Agricultural and Resource Economics Review, 41(1):114123, 2012. doi: 10.1017/S1068280500004238

[38] W. Hedgcock and A. R. Rao. Trade-off aversion as an explanation for the attraction effect: A functional magnetic resonance imaging study. Journal of Marketing Research, 46(1):1-13, 2009.

[39] W. Hedgcock, A. R. Rao, and H. A. Chen. Could ralph nader's entrance and exit have helped al gore? the impact of decoy dynamics on consumer choice. Journal of Marketing Research, 46(3):330-343, 2009.

[40] I. Hernandez and J. L. Preston. Disfluency disrupts the confirmation bias. Journal of Experimental Social Psychology, 49(1):178-182, 2013.

[41] S. Highhouse. Context-dependent selection: The effects of decoy and phantom job candidates. Organizational Behavior and Human Decision Processes, 65(1):68 - 76, 1996.

[42] R. M. Hogarth. A note on aggregating opinions. Organizational behavior and human performance, 21(1):40-46, 1978.

[43] J. Huber, J. W. Payne, and C. Puto. Adding asymmetrically dominated alternatives: Violations of regularity and the similarity hypothesis. Journal of Consumer Research, 9(1):90-98, 1982.

[44] J. Huber, J. W. Payne, and C. P. Puto. Let's be honest about the attraction effect. Journal of Marketing Research, 51(4):520-525, 2014.

[45] J. Hullman, P. Resnick, and E. Adar. Hypothetical outcome plots outperform error bars and violin plots for inferences about reliability of variable ordering. PloS one, 10(11):e0142444, 2015.

[46] P. Isenberg, D. Fisher, M. R. Morris, K. Inkpen, and M. Czerwinski. An exploratory study of co-located collaborative visual analytics around a tabletop display. In Visual Analytics Science and Technology (VAST), 2010 IEEE Symposium on, pp. 179-186. IEEE, 2010.

[47] K. Jenni and G. Loewenstein. Explaining the identifiable victim effect. Journal of Risk and Uncertainty, 14(3):235-257, 1997.

[48] A. Jiménez, S. Rios-Insua, and A. Mateos. A decision support system for multiattribute utility evaluation based on imprecise assignments. Decision Support Systems, 36(1):65-79, 2003.

[49] D. Kahneman. A perspective on judgment and choice: mapping bounded rationality. American psychologist, 58(9):697, 2003.

[50] D. Kahneman. Thinking, fast and slow. Macmillan, 2011.

[51] D. Kahneman and A. Tversky. Subjective probability: A judgment of representativeness. Cognitive psychology, 3(3):430-454, 1972.

[52] D. Kahneman and A. Tversky. On the reality of cognitive illusions. 1996.

[53] J. Klayman and K. Brown. Debias the environment instead of the judge: 
An alternative approach to reducing error in diagnostic (and other) judgment. Cognition, 49(1):97-122, 1993.

[54] R. P. Larrick, J. N. Morgan, and R. E. Nisbett. Teaching the use of costbenefit reasoning in everyday life. Psychological Science, 1(6):362-370, 1990.

[55] T. Latty and M. Beekman. Irrational decision-making in an amoeboid organism: transitivity and context-dependent preferences. Proc. of the Royal Society B: Biological Sciences, 278(1703):307, 2011.

[56] D. R. Lehman and R. E. Nisbett. A longitudinal study of the effects of undergraduate training on reasoning. Developmental Psychology, 26(6):952, 1990.

[57] I. S. MacKenzie. Fitts' law as a research and design tool in humancomputer interaction. Human-computer interaction, 7(1):91-139, 1992.

[58] R. M. Martey, A. Shaw, J. Stromer-Galley, K. Kenski, B. A. Clegg, J. E. Folkestad, E. T. Saulnier, and T. Strzalkowski. Testing the power of game lessons: The effects of art and narrative on reducing cognitive biases. In DiGRA, 2014.

[59] L. Micallef, P. Dragicevic, and J.-D. Fekete. Assessing the effect of visualizations on bayesian reasoning through crowdsourcing. IEEE Transactions on Visualization and Computer Graphics, 18(12):2536-2545, 2012.

[60] C. K. Morewedge, H. Yoon, I. Scopelliti, C. W. Symborski, J. H. Korris, and K. S. Kassam. Debiasing decisions: Improved decision making with a single training intervention. Policy Insights from the Behavioral and Brain Sciences, 2(1):129-140, 2015.

[61] M. Mortell, H. H. Balkhy, E. B. Tannous, and M. T. Jong. Physician defiance towards hand hygiene compliance: Is there a theory-practiceethics gap? Journal of the Saudi Heart Association, 25(3):203-208, 2013.

[62] S. Moscovici and M. Zavalloni. The group as a polarizer of attitudes. Journal of personality and social psychology, 12(2):125, 1969.

[63] T. Munzner. Visualization Analysis and Design. CRC Press, 2014.

[64] T. Mussweiler, F. Strack, and T. Pfeiffer. Overcoming the inevitable anchoring effect: Considering the opposite compensates for selective accessibility. Personality and Social Psychology Bulletin, 26(9):11421150, 2000.

[65] J. Navajas, T. Niella, G. Garbulsky, B. Bahrami, and M. Sigman. Deliberation increases the wisdom of crowds. preprint arXiv:1703.00045, 2017.

[66] R. S. Nickerson. Confirmation bias: A ubiquitous phenomenon in many guises. Review of general psychology, 2(2):175, 1998.

[67] Y. P. S. O'Curry and R. Pitts. The attraction effect and political choice in two elections. Journal of Consumer Psychology, 4(1):85-101, 1995.

[68] A. Ottley, E. M. Peck, L. T. Harrison, D. Afergan, C. Ziemkiewicz, H. A. Taylor, P. K. Han, and R. Chang. Improving bayesian reasoning: The effects of phrasing, visualization, and spatial ability. IEEE transactions on visualization and computer graphics, 22(1):529-538, 2016.

[69] R. O. Ottosson, P. E. Engström, D. Sjöström, C. F. Behrens, A. Karlsson, T. Knöös, and C. Ceberg. The feasibility of using pareto fronts for comparison of treatment planning systems and delivery techniques. Acta Oncologica, 48(2):233-237, 2009.

[70] S. Pajer, M. Streit, T. Torsney-Weir, F. Spechtenhauser, T. Muller, and H. Piringer. Weightlifter: Visual weight space exploration for multicriteria decision making. IEEE transactions on visualization and computer graphics, 23(1):611, 2017.

[71] A. V. Pandey, K. Rall, M. L. Satterthwaite, O. Nov, and E. Bertini. How deceptive are deceptive visualizations?: An empirical analysis of common distortion techniques. In ACM Conference on Human Factors in Computing Systems, pp. 1469-1478. ACM, 2015.

[72] J. W. Payne, J. R. Bettman, and E. J. Johnson. The adaptive decision maker. Cambridge University Press, 1993.

[73] J. C. Pettibone and D. H. Wedell. Examining models of nondominated decoy effects across judgment and choice. Organizational Behavior and Human Decision Processes, 81(2):300 - 328, 2000.

[74] S. Plous. The psychology of judgment and decision making. Mcgraw-Hill Book Company, 1993.

[75] R. F. Pohl. Cognitive illusions: Intriguing Phenomena in thinking, judgement and memory. Psychology Press, 2016.

[76] D. Prelec, H. S. Seung, and J. McCoy. A solution to the single-question crowd wisdom problem. Nature, 541(7638):532-535, 2017.

[77] V. F. Reyna, C. F. Chick, J. C. Corbin, and A. N. Hsia. Developmental reversals in risky decision making: Intelligence agents show larger decision biases than college students. Psychological science, 25(1):76-84, 2014.

[78] S. F. Roth and J. Mattis. Data characterization for intelligent graphics presentation. In Proc. of the SIGCHI Conference on Human Factors in Computing Systems, pp. 193-200. ACM, 1990.

[79] L. J. Sanna, N. Schwarz, and S. L. Stocker. When debiasing backfires: accessible content and accessibility experiences in debiasing hindsight. Journal of Experimental Psychology: Learning, Memory, and Cognition, 28(3):497, 2002.

[80] H. A. Simon. Rational choice and the structure of the environment. Psychological review, 63(2):129, 1956.

[81] I. Simonson. Choice based on reasons: The case of attraction and compromise effects. Journal of Consumer Research, 16(2):158-174, 1989.

[82] I. Simonson and A. Tversky. Choice in context: Tradeoff contrast and extremeness aversion. Journal of Marketing Research, 29:281-295, 1992.

[83] J. B. Soll and R. P. Larrick. Strategies for revising judgment: How (and how well) people use others opinions. Journal of Experimental Psychology: Learning, Memory, and Cognition, 35(3):780, 2009.

[84] E. Soyer and R. M. Hogarth. The illusion of predictability: How regression statistics mislead experts. International Journal of Forecasting, 28(3):695711, 2012.

[85] R. R. Springmeyer, M. M. Blattner, and N. L. Max. A characterization of the scientific data analysis process. In Proc. of the 3rd conference on Visualization'92, pp. 235-242. IEEE Computer Society Press, 1992.

[86] A. Tversky. Elimination by aspects: A theory of choice. Psychological Review, 79:281-299, 1972.

[87] A. C. Valdez, M. Ziefle, and M. Sedlmair. Priming and anchoring effects in visualizations. IEEE Transactions on Visualization and Computer Graphics, 2017.

[88] J. L. Voss, K. D. Federmeier, and K. A. Paller. The potato chip really does look like elvis! neural hallmarks of conceptual processing associated with finding novel shapes subjectively meaningful. Cerebral Cortex, 22(10):2354-2364, 2011.

[89] E. Wall, L. M. Blaha, L. Franklin, and A. Endert. Warning, bias may occur: A proposed approach to detecting cognitive bias in interactive visual analytics. In IEEE Conference on Visual Analytics Science and Technology (VAST), 2017.

[90] D. H. Wedell. Distinguishing among models of contextually induced preference reversals. Journal of Experimental Psychology: Learning, Memory, and Cognition, 17(4):767, 1991.

[91] S. Wehrend and C. Lewis. A problem-oriented classification of visualization techniques. In Proc. of Visualization'90, pp. 139-143. IEEE Computer Society Press, 1990.

[92] R. F. West, R. J. Meserve, and K. E. Stanovich. Cognitive sophistication does not attenuate the bias blind spot. Journal of personality and social psychology, 103(3):506, 2012.

[93] H. Wickham, D. Cook, H. Hofmann, and A. Buja. Graphical inference for infovis. IEEE Transactions on Visualization and Computer Graphics, 16(6):973-979, 2010.

[94] P. Wright. Consumer choice strategies: Simplifying vs. optimizing. Journal of Marketing Research, pp. 60-67, 1975.

[95] J. S. Yi, Y. ah Kang, and J. Stasko. Toward a deeper understanding of the role of interaction in information visualization. IEEE transactions on visualization and computer graphics, 13(6):1224-1231, 2007.

[96] J. C. Zhao, W.-T. Fu, H. Zhang, S. Zhao, and H. Duh. To risk or not to risk?: Improving financial risk taking of older adults by online social information. In ACM Conference on Computer Supported Cooperative Work \& Social Computing, pp. 95-104. ACM, 2015.

[97] B. J. Zikmund-Fisher, A. M. Scherer, H. O. Witteman, J. B. Solomon, N. L. Exe, and A. Fagerlin. Effect of harm anchors in visual displays of test results on patient perceptions of urgency about near-normal values: Experimental study. Journal of medical Internet research, 20(3), 2018.

[98] T. Zuk and S. Carpendale. Visualization of uncertainty and reasoning. In Intern. Symposium on Smart Graphics, pp. 164-177. Springer, 2007. 